\title{
Téoros
}

Revue de recherche en tourisme

\section{Qualité et classification} Des enjeux d'importance

\section{Normand Cazelais}

Volume 23, numéro 2, été 2004

La qualité en tourisme

URI : https://id.erudit.org/iderudit/1071305ar

DOI : https://doi.org/10.7202/1071305ar

Aller au sommaire du numéro

Éditeur(s)

Université du Québec à Montréal

ISSN

0712-8657 (imprimé)

1923-2705 (numérique)

Découvrir la revue

\section{Citer cet article}

Cazelais, N. (2004). Qualité et classification : des enjeux d'importance. Téoros, 23(2), 34-37. https://doi.org/10.7202/1071305ar d'utilisation que vous pouvez consulter en ligne.

https://apropos.erudit.org/fr/usagers/politique-dutilisation/ 


\title{
Qualité et classification
}

\author{
Des enjeux d'importance
}

\section{Normand Cazelais}

En tourisme, comme dans bien d'autres domaines, la qualité est souvent affaire de classification. Toute classification induit une cotation. Ainsi, la réputation d'un établissement dépendra largement de l'échelon où il s'inscrit dans la gamme des évaluations, qu'elles soient officielles ou officieuses.

Par classification, le Robert entend « l'action de distribuer par classes, par catégories » et le «résultat de cette action». Il définit ainsi le mot classe: "grade, rang concernant l'importance, la valeur, la qualité ». Pour établir cette importance, cette valeur, cette qualité, toute classification recourt donc à des critères, «à ce qui sert de base à un jugement ». Or, selon l'objectif visé ou la nature de la classification recherchée, le choix et l'application des critères sont éminemment variables.

\section{Classification et classifications}

En tourisme, on peut distinguer trois grands groupes de classifications.

\section{La classification officielle}

La classification dite officielle qui est habituellement celle de l'État ou de l'un de ses mandataires. Comme la France, la Grande-Bretagne, le Brésil ou toute structure politique de cet ordre dûment organisée, le Québec a édicté une classification hôtelière qui touche deux types d'établissements :

Les soleils sont utilisés exclusivement dans la classification des gîtes touristiques, alors que les étoiles sont attribuées aux hôtels, résidences de tourisme, centres de vacances, auberges de jeunesse et établissements d'enseignement. La classification hôtelière est sous la responsabilité de la Corporation de l'industrie touristique du Québec (CITQ), qui évalue tous les établissements d'hébergement $d u$ Québec selon des critères bien précis'.

Le tableau 1 présente la classification des établissements d'hébergement (hôteliers et gites), basée sur des normes et des critères internationaux ${ }^{2}$ et établie par le programme Hébergement Québec lui-même «mis en auvre par l'industrie touristique en collaboration avec Tourisme Québec ${ }^{3} »$.

Nous ne procéderons pas dans ce texte à l'analyse exhaustive de critères; il y aurait là le sujet d'un livre en entier. Qu'il nous suffise de souligner qu'ils s'apparentent, par exemple, à ceux du Bénélux ${ }^{4}$, c'est-à-dire qu'ils s'appuient sur une appréciation «objective » d'un certain nombre d'éléments tangibles (mobilier, sanitaires et salles de bains, accès et circulation, locaux et services dispensés tels que parking, restauration, blanchisserie, etc.) ou autrement matériels (entretien, hygiène et propreté, etc.).

Évidemment, les critères et les normes ne font pas l'unanimité et nombre d'exploitants, sinon de clients, déplorent qu'ils ne rendent pas toujours justice aux établissements ainsi classifiés puisque, dans une telle approche, des éléments comme le site, le paysage, le milieu ambiant, la décoration intérieure ont un moindre poids dans la balance.

Cependant, quels que soient ses limites ou ses défauts, la classification officielle joue un rôle significatif et reconnu dans la confirmation et la valorisation d'un produit touristique en particulier et du produit touristique en général. C'est ce que nous verrons ci-dessous, pour le Québec du moins.

\section{La classification des guides}

Tant l'État que les exploitants touristiques et les voyageurs eux-mêmes ont, depuis qu'elle existe, salué l'utilité d'une classification pourtant officieuse qu'est celle des guides touristiques. Baedeker le fait depuis

\section{TABLEAU 1 : Établissements d'hébergement touristique - Pannonceaux des gites et des hôtels}

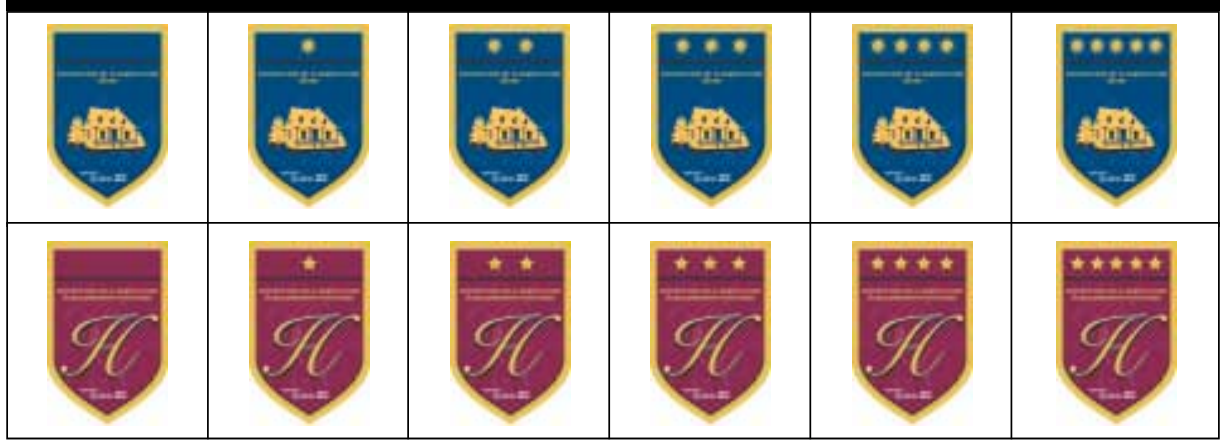

Source : Corporation de l'industrie touristique du Québec (CITQ). 
le dix-neuvième siècle en Allemagne, tout comme Ulysse chez nous depuis une vingtaine d'années : ces ouvrages accordent, comme manifestation de leur compétence et de leur autorité, des cotes - sous forme d'étoiles, par exemple - à des établissements de restauration et d'hébergement ou même parfois à des sites, à des localités, à des circuits et à des régions entières. De cette manière, ils attestent d'une qualité par des sceaux qui correspondent aux valeurs spécifiques que chaque guide véhicule.

Le cas du guide Michelin est sûrement le plus connu. L'entreprise de Clermont-Ferrand a eu du flair, sinon du génie, en offrant « gracieusement»-car «il ne doit pas être vendu », précisait-elle en quatrième de couverture - aux chauffeurs et vélocipédistes son premier Guide rouge en 1900. Dans l'avant-propos, on pouvait lire :

Cet ouvrage paraît avec le siècle; il durera autant que lui. L'automobilisme vient de naître; il se développera chaque année et le pneu avec lui. [...] Chaque année nous publierons une nouvelle édition mise soigneusement à jour. [...] L'ouvrage se perfectionnera d'année en année; il sera parfait d'autant plus vite que les chauffeurs répondront plus soigneusement et en plus grand nombre au questionnaire que nous les prions de vouloir bien remplir ${ }^{5}$.

Cent ans plus tard, ces espoirs ont été plus que comblés. Certes, les automobilistes ont répondu à l'appel, mais, mieux encore, l'attribution de macarons aux tables dans le Guide rouge et d'étoiles aux sites, aux circuits et aux régions dans le Guide vert a fait de Michelin une référence incontestée pendant des générations, d'abord dans l'Hexagone et, par la suite, dans un grand nombre de destinations. Sans compter que la réputation des guides a eu des répercussions non négligeables sur la prospérité du fabricant de pneumatiques...

Fait à signaler, contrairement aux responsables de la classification officielle, les maisons d'édition de guides et leurs auteurs n'ont pas à dévoiler et encore moins à expliquer leurs critères de classification. À leurs yeux, la confirmation par le public - et aussi, dans une certaine mesure, par les professionnels - de la justesse de leurs évaluations en constitue le meilleur barème.

Les classifications officieuses des diverses familles de guides, quelle que soit la langue, se sont partout révélées un puissant contrepoids aux classifications officielles, parce que le public en général et les voyageurs en particulier y ont trouvé et y trouvent toujours une autre vision, un autre point de vue, qui peuvent se permettre davantage de subjectivité. Cela explique par ailleurs que l'autorité des guides touristiques puisse certaines fois être remise en question, ce que nous constaterons ci-après.

\section{Les classifications internes}

Un autre type de classification officieuse qui exerce une forte influence sur une attestation de qualité relève des entreprises elles-mêmes ou, parfois, des destinations : retenons pour exemples l'Association des beaux villages du Québec et le tout récent Club des plus belles baies du monde qui a tenu en 2003 son deuxième congrès mondial à Tadoussac.

Mais restons dans le domaine de l'hébergement : en adhérant à une bannière, des établissements indépendants expriment leurs objectifs d'affaires, les créneaux visés et les caractéristiques de leurs produits, sans renoncer à leur autonomie. Leading Hotels of the World, Les Relais du Silence, Hotels Romantik sont, dans cet esprit, autant de cartes d'identité et de labels de qualité.

Le groupe Relais \& Châteaux est éloquent à ce propos. Voilà cinquante ans, le 12 mai 1954, était signé son acte de naissance. À l'initiative de Marcel et Nelly Tilloy, alors propriétaires de l'Hostellerie La Cardinale, établissement de sept chambres à Baix, huit maisons situées sur l'axe Paris-Nice se sont unies pour créer les Relais de Campagne : c'était la « Route du Bonheur ».

Pour en faire partie, ces maisons devaient être «silencieuses, agréablement meublées, présenter des jardins entretenus, un accueil parfait et avoir une bonne table ${ }^{6} \gg$. Deux ans plus tard, elles étaient déjà vingt-cinq ré- parties dans toute la France et, en 1960 alors que germait le Marché Commun, quinze nouveaux adhérents les rejoignaient. Les formules qui ont du succès suscitent des émules : en 1962, apparaît un concurrent, la chaîne des Châteaux-Hôtels et, dix ans après, naissent les Relais Gourmands, étapes gastronomiques vite très renommées.

Le reste appartient à l'histoire : les Relais de Campagne, Châteaux-Hôtels et Relais Gourmands fusionnent et deviennent les Relais \& Châteaux en 1975. Une histoire qui s'accélère: le groupe rassemble 290 établissements dans 23 pays en 1979 et 375 dans 36 contrées sur cinq continents en $1989^{7}$. Élue, cette année-là, la «plus belle du monde » par ses clients, la chaîne devient un symbole de qualité hôtelière à travers le monde.

Mais un tel résultat n'est pas évident. Depuis la fin de la Seconde Guerre mondiale, le monde du voyage en général et celui de l'hôtellerie en particulier sont dominés par de vastes conglomérats organisationnels et financiers; dans un tel contexte, faire sa place au soleil, surtout dans les créneaux supérieurs, n'est pas une mince affaire pour une petite entreprise individuelle.

La raison d'être des Relais \& Châteaux est d'occuper les créneaux supérieurs et de regrouper, pour ce faire, des propriétaires d'hôtels ou de restaurants aptes à répondre aux critères de sa « charte de qualité » que sont les cinq $\mathrm{C}$ : caractère, courtoisie, calme, charme, cuisine. Pour accentuer son image de marque, la chaîne privilégie la personnalisation de l'accueil et un service sur mesure favorisés par des établissements de petite taille (25 chambres en moyenne). Elle veut aussi mettre l'accent plus sur le charme que sur le luxe, affirmant que «le charme n'a pas de prix, qu'au contraire du luxe, il n'est jamais facturé ${ }^{8} »$. Autant de critères, on l'aura compris, qu'on ne peut guère assimiler à des éléments comptabilisables.

Une autre approche utilisée par l'industrie pour procéder à une classification officieuse est celle des grandes chaînes hôtelières qui créent différentes bannières au sein de leurs groupes respectifs pour 
occuper le plus de champ possible dans les divers créneaux. Voyons brièvement les stratégies du Groupe Accor et de Marriott International. Le premier, né en France, compte près de 4000 établissements dans 140 pays, emploie 158000 personnes et a réalisé en 2002 un chiffre d'affaires de 7,1 milliards d'euros. De taille plus réduite (2400 établissements), le second, originaire des États-Unis, fait tout de même partie des grandes chaînes hôtelières mondiales.

Tous deux ont une stratégie similaire: ils déclinent leurs établissements sous différents visages (identifiés à des bannières) pour rejoindre, en fonction de leurs besoins et leurs attentes spécifiques, des clientèles variées et le plus grand nombre de voyageurs possible. L'éventail Accor va de l'économique au luxe, des Hôtels Formule 1 aux Sofitel en passant par les Novotel, Coralia, Accor Thalassa, Mercure, Atria, Accor Suitehôtel, Ibis, Etap, Red Roof Inns, Studio 6 et Motel 6. Davantage orientée vers le moyen et le haut de gamme, la panoplie de Marriott comprend Marriott Hotels \& Resorts, JW Marriott Hotels \& Resorts, Renaissance Hotels \& Resorts, Courtyard, Residence Inn, Fairfield Inn, Towne Place Suites, SpringHill Suites, Marriott Vacation Club International et Marriott Conference Centers.

Pour l'une et l'autre des compagnies, chaque bannière a ses caractéristiques propres qui correspondent à des critères d'aménagement et de gestion précis ${ }^{9}$. En conséquence, ces bannières identifient des niveaux de services, d'atmosphère et de luxe et toutes doivent constituer, dans leurs sphères respectives, des labels de qualité. Ici, la classification prend donc un autre sens.

\section{Incidences et conséquences}

\section{Le constat québécois}

Le 16 avril 2004, Nathalie Normandeau, ministre déléguée au Développement régional et au Tourisme, et Jean Authier, président de la Corporation de l'industrie touristique du Québec (CITQ), dévoilent les résultats d'une tournée de classification des établissements d'hébergement touristique du Québec menée en 2002 et 2003 par la
CITQ, organisme sans but lucratif mandaté pour ce faire par Tourisme Québec. Première en son genre depuis l'adoption de la Loi sur les établissements d'hébergement touristique en juillet 2000, elle a couvert sept des neuf catégories concernées : les établissements hôteliers, les gîtes, les résidences de tourisme, les centres de vacances, les villages d'accueil, les auberges de jeunesse et les établissements d'enseignement.

Le constat formulé par les deux partenaires se résume ainsi: le Québec dispose d'une grande diversité d'établissements dans la plupart des régions et, mieux encore, d'un solide noyau d'établissements de fort calibre. En effet, au terme de la tournée, 23 établissements hôteliers reçoivent la cote maximale, à savoir cinq étoiles, et 22 gîtes reçoivent l'équivalent, soit cinq soleils.

Manifestement, les résultats (voir tableau 2) dépassent le cadre d'une simple opération de classification. La ministre déclare alors : "[Ces] résultats [...] indiquent que l'industrie a fait, en quelques années à peine, des gains appréciables sur le plan de la qualité du produit ${ }^{10}$. » Si elles sont objectivement conformes à la réalité, ces conclusions n'en demeurent pas moins marquées par l'optimisme, puisque que, de 2002 à 2003, ce ne sont que 334 de 5237 établissements qui ont vu leur niveau de classification augmenter alors que 91 autres ont accusé une rétrogradation.
N'empêche, aux yeux du président de la CITQ, ces «progrès » sont une «preuve éloquente de la volonté et du dynamisme» de l'industrie touristique et de son «engagement envers la qualité ». Aussi, précise-t-il «Ces transformations se font sur une toile de fond dominante : l'instauration d'un programme officiel de classification a suscité une prise de conscience de plus en plus aiguë chez les propriétaires d'établissements que l'hébergement de qualité est désormais un atout majeur, un atout sur lequel il faudra miser au cours des prochaines années ${ }^{11}$. »

\section{Les misères de Michelin}

Les confidences Pascal Rémy, en février dernier, de dans une entrevue choc accordée à l'influent Figaro Magazine ${ }^{13}$ ont l'effet d'une bombe. En France, bien sûr, mais aussi ailleurs. Ainsi, en prenant le relais de l'Associated Press quelques jours plus tard, le Miami Herald souligne que le Guide rouge Michelin, «étalon de la gastronomie française ${ }^{13} »$, vacille sur ses bases.

Cet ex-inspecteur de l'auguste maison, licencié parce qu'il comptait publier des carnets résumant son expérience de près de vingt ans en la matière, révèle entre autres que, pour établir ses jugements qui font -et défont - fortunes et réputations, Michelin n'a recours qu'à un nombre restreint d'enquêteurs «confirmés » (cinq en fait en 2003), que l'influence du marketing est

\begin{tabular}{|c|c|c|c|c|c|c|c|}
\hline \multirow{2}{*}{ Établissements touristiques } & \multicolumn{7}{|c|}{ Niveaux de classification } \\
\hline & 0 & 1 & 2 & 3 & 4 & 5 & Total \\
\hline$\star$ Établissements hôteliers & 46 & 405 & 757 & 553 & 169 & 23 & 1953 \\
\hline Gîtes & 0 & 13 & 202 & 896 & 440 & 22 & 1573 \\
\hline$\star$ Résidences de tourisme & 8 & 79 & 419 & 748 & 113 & & 1367 \\
\hline$\star$ Centres de vacances & 3 & 22 & 86 & 113 & 34 & & 258 \\
\hline$\star$ Villages d'accueil & 0 & 1 & 1 & 9 & 3 & & 14 \\
\hline$\star$ Auberges de jeunesse & 0 & 8 & 20 & 16 & & & 44 \\
\hline$\star$ Établissements d'enseignement & 0 & 3 & 19 & 6 & & & 28 \\
\hline Total & 57 & 531 & 1504 & 2341 & 759 & 45 & 5237 \\
\hline
\end{tabular}

Niveau de classification inexistant dans cette catégorie Source : CITQ en date du 12 avril 2004 
toute puissante, qu'il y a des chefs surévalués et même des intouchables chez les trois étoiles. Bref, affirme-t-il en substance, il faut s'interroger sur la démarche et l'évolution d'un guide, qualifié de «terrible » par certains, qui fait autorité en cuisine depuis plus de trois générations.

À la même période, Le Monde, très sérieux quotidien de l'Hexagone, parle déjà au passé : «Le Michelin, c'était la conscience subliminale des Français. Il ressemblait de loin à une société secrète. Tourisme, gastronomie et culture étaient le jeu du Michelin. » À peine étonné de la situation, le quotidien n'était cependant «pas prêt à dire » qu'il est devenu un guide «comme les autres» parce qu'il «jouit encore de la confiance de ses lecteurs». Mais «Pour longtemps $^{14}$ ? $\gg$ demande-t-il.

À l'origine, soulignons-le, le guide Michelin était un outil mis à la disposition des voyageurs qui, avec leurs chevaux-vapeur, osaient s'aventurer sur des routes mal entretenues et en des lieux souvent dotés de services de mécanique, d'hébergement et de restauration déficients. Tout en jouant ce rôle, il a été également un formidable outil de marketing. Qui aujourd'hui ne connaît le Bibendum?

Tout comme les guides, les pneus Michelin ont imposé une réputation de qualité. Et c'est là, curieusement, que le bât blesse : Pascal Rémy confie au Figaro Magazine

parfois, le Michelin se méfie de retentissements trop forts pouvant nuire à son image. Il a créé des chefs médiatiquement plus puissants que lui. Sa marge de manœuvre est réduite d'autant. L'obsession, c'est la réduction des coûts et la diversification des produits. Toute publication Michelin doit aujourd'hui rapporter de l'argent. [...] Depuis la création du service marketing, cet aspect prend de l'ampleur de semaine en semaine. Le directeur général du guide est également le directeur du service marketing du guide ${ }^{15}$.

D'où la question du Monde: «À qui se fier ${ }^{16}$ ?»

\section{L'emprise et l'empire du marketing}

En parallèle à la classification officielle des États et à la classification officieuse des guides touristiques, les grandes chaînes hôtelières procèdent, on l'a vu, à leurs propres classifications internes. Plus encore, ils les publicisent largement, au moyen de répertoires-maisons et par des insertions dans les brochures des grossistes et de voyagistes, pour convaincre les consommateurs de la qualité de leur offre, quels que soient le niveau de luxe ou le type de prestations des différents établissements ainsi présentés. Ce que font notamment le Groupe Accor et Marriott International.

Cette classification intra-muros a un grand avantage : elle n'a pas besoin de se justifier. Sa force de conviction s'appuie sur un outil qui n'appartient pas à la grande famille des critères. Cet outil a un nom : le marketing.

Si les classifications officielle et officieuse citées ci-haut définissent des niveaux de qualité à l'aide de critères génériques, les grandes chaînes hôtelières ont recours, quant à elles, à des armes qui se révèlent aussi, sinon plus, efficaces et persuasives : leurs diverses catégories d'établissements participent en effet à la promotion d'images et de symboles segmentés avec soin pour persuader les voyageurs d'opter pour ces produits en fonction de leurs attentes et de leurs besoins qui peuvent varier dans le temps et dans l'espace. Il ne s'agit plus, dans ce cas, de faire savoir si l'établissement $\mathrm{ABC}$ vaut vraiment telle ou telle cote, mais de persuader les consommateurs qu'il est - intrinsèquement - de qualité et qu'il saura les combler quand il le faudra.

Dans cette logique, attirer et convaincre ne suffisent pas, il importe en outre de fidéliser, c'est-à-dire d'assurer un taux de retour qui devient, en quelque sorte, la démonstration et le prolongement de la classification interne initiale. C'est alors qu'entrent en action les clubs d'adhérents qui garantissent une panoplie d'avantages et les banques de points dont l'accumulation promet aux détenteurs des avantages analogues ou complémentaires ${ }^{17}$.

Un tel système, dont l'efficacité n'est plus à démontrer, permet aux entreprises qui les utilisent d'occuper des parts de marché sans cesse plus considérables et de créer des tendances à leur profit, car la spirale des avantages consentis aux voyageurs rétrécit, dans les faits, leurs choix. En d'autres mots, il leur permet d'influer sur le développement touristique en fonction de leurs objectifs internes tout en proposant des modèles de qualité et en modelant, du même coup, les comportements des consommateurs.

En termes de résultats, la subjectivité avouée de cette approche l'emporte sur l'objectivité des critères de classification.

Normand Cazelais est chroniqueur en tourisme au journal Le Devoir.

\section{Notes}

1 Voir le Nouveau programme de classification des établissements hôteliers du Québec [http://www.bonjourquebec.com/francais/ general/faq/pratique.html\#15].

2 «Les critères d'évaluation utilisés pour classifier les établissements hôteliers ont été établis à la suite d'une vaste consultation auprès d'hôteliers, d'experts en classification hôtelière de Tourisme Québec et de représentants de l'industrie touristique. Des recherches ont également été effectuées auprès d'organismes expérimentés en classification hôtelière. Les programmes de classification des Fleurs-de-lys de Tourisme Québec ainsi que ceux appliqués en France et en Suisse ont également guidé le groupe de travail dans ses démarches. » (Nouveau programme de classification des établissements hôteliers du Québec, Hébergement Québec : Classification hôtelière [www.airservice.com/rustique/clas/html].

3 Voir [www.gite.ca/hebergementquebec/ francais/hebergement/classif.html].

4 Classification hôtelière Benelux [www.mdt.public.lu/classification/hotellerie/ classification_hoteliere_benelux.doc].

5 Guide rouge Michelin, $1^{\mathrm{re}}$ édition, 1900, Paris.

6 Voir le site Internet [www.relaischateaux.com].

7450 établissements dans 50 pays sur cinq continents en 2004.

8 Consulter le site [www.relaischateaux.com].

9 Consulter les sites [www.accor.com] et [http://marriott.com].

10 Source personnelle.

11 Idem.

12 Le Figaro Magazine, le 14 février 2004.

13 The Miami Herald, le 19 février 2004.

14 Le Monde, le 20 février 2004.

15 Le Figaro Magazine, le 14 février 2004.

16 Le Monde, le 20 février 2004.

17 Lire à cet effet le communiqué émis le 19 avril 2004 par le groupe hôtelier canadien Fairmont Hotels \& Resorts [fairmont2 fairmontmarketing.com] intitulé Merci de votre fidélité - Voici les nouveaux privilèges du Club Président des Hôtels Fairmount. 